\title{
Synthesis, Spectral and Antimicrobial Studies of Some N(2)-Substituted Tetrahydroindazoles
}

\author{
Shanmugasundaram Amirthaganesan, Gopalakrishnan Aridoss, Keun Soo Park, Kwon Taek Lim, and Yeon Tae Jeong \\ Division of Image Science and Information Engineering, Pukyong National University, Busan 608-739, Korea \\ *E-mail: ytjeong@pknu.ac.kr \\ Received November 25, 2009, Accepted February 22, 2010
}

\begin{abstract}
A series of N(2)-benzothiazolyl substituted tetrahydroindazoles has been synthesized via cyclic $\beta$ keto esters. Optimum reaction condition was found as acidic toluene and effect of higher acidity towards substituted hydrazines in situ was described. Synthesized compounds have been achieved as single isomer and characterized by using 1D and 2D NMR spectral reports. Antimicrobial screening was carried out for the synthesized compounds along with a series of $N(2)$-pyridyl tetrahydroindazoles. ${ }^{1}$ The results of the in vitro antimicrobial screening studies revealed that compounds 13, 16 against Staphylococcus aureus, 11 against Escherichia coli, 10-12, 16 against Pseudomonas aeruginosa and 12 against Klebsiella pneumoniae recorded almost two-fold better activity compared to the standard drug used.
\end{abstract}

Key Words: Tetrahydroindazoles, Benzothiazolyl, 1(H) Indazoles, Antimicrobial activity

\section{Introduction}

It is noteworthy to record the microbial evaluation of organic compounds for the development of current research in drug discovery and medicinal chemistry. Azole compounds possess a broad history in the field of heterocyclic compounds due to their wide range of applications. Pyrazoles, ${ }^{2}$ the well known azole based skeleton are focusing to explore their biological potencies. ${ }^{3}$ Moreover, substituted pyrazoles and pyrazole derivatives such as benzopyrazoles ${ }^{4}$ (aromatic derivatives of pyrazoles) and tetrahydroindazoles ${ }^{5}$ (cycloalkane derivatives of pyrazoles) along with several substituents are having much attention for their unique pharmacological applications. ${ }^{6-8} \mathrm{Ge}-$ nerally, pyrazoles and substituted pyrazoles have been synthesized by the reactions of hydrazines with $\beta$-dicarbonyl compounds ${ }^{9}$ and tetrahydroindazoles have been synthesized from cyclohexanones and its derivatives. ${ }^{10}$ Several reports described about the annular tautomersim ${ }^{11}$ of pyrazoles and formation of regioisomers ${ }^{12}$ in unsymmetrical pyrazoles and tetrahydroindazoles during the $N$-substitution like $N$-arylation ${ }^{13}$ and $\mathrm{N}$-alkylation. ${ }^{14}$ Even though there are several methods, it is in need of creating general methodologies for the regioselective synthesis of biologically potent $N$-substituted tetrahydroindazoles with various substituents in the saturated ring.

\section{Experimental Section}

The progress of the reactions and purity of the products were ascertained by performing TLC. Melting points were determined in Electrothermal-9100 (Japan) instrument and are uncorrected. All the NMR spectra were recorded on JEOL (Japan) JNM ECP-400 instrument operating at $400 \mathrm{MHz}$ for ${ }^{1} \mathrm{H}$ and 100.6 MHz for completely proton decoupled ${ }^{13} \mathrm{C}$. The tubes used for recording NMR spectra are of $5 \mathrm{~mm}$ in diameter and DMSO- $d_{6}$ was used as solvent (for compound $\mathbf{2 8}, \mathrm{CDCl}_{3}+$ DMSO- $d_{6}$ was used). The chemical shift values are reported in ppm (parts per million) relative to TMS and coupling con- stant $(J)$ values are represented in $\mathrm{Hz}$ (Hertz). Aromatic protons of phenyl group at $\mathrm{C}-4$ are mentioned as $\mathrm{Ph}-\mathrm{H}$ and protons of benzothizole moiety are given as BTz-H. Microanalyses were performed on Heraeus Carlo Erba 1108 CHN analyzer.

General procedure for the synthesis of r(2),c(4)-bis(alkoxycarbonyl)- $t(3)$-aryl-c(5)-hydroxy- $t$ (5)-methylcyclohexanones (1-9). A mixture of acetoacetic ester $(10 \mathrm{mmol})$, aromatic aldehyde $(5 \mathrm{mmol})$ and methylamine $(5 \mathrm{mmol})$ in ethanol was heated to boiling. The reaction mixture was kept for overnight. The separated solid was filtered and recrystalized from ethanol. ${ }^{15}$

General procedure for the synthesis $r(4)$-aryl-t(5)-alkoxycarbonyl-t(6)-hydroxy-c(6)-methyl-4,5,6,7-tetrahydro-3-hydroxy-2-(benzothiazol-2-yl)-indazoles (10-18). A mixture of cyclic ketoester $(1 \mathrm{mmol})$ and 2-hydrazinobenzothiazole (1.2 $\mathrm{mmol}$ ) in toluene with the catalytic amount of acetic acid was refluxed for about 3 - $5 \mathrm{hrs}$. Duration of the reaction varied for each reaction, and it was monitored by TLC. After completion of the reaction, the solvent was removed under vacuum and the resultant residue was recrystalized from ethanol.

Synthesis of $t(5)$-methoxycarbonyl-t(6)-hydroxy-c(6)-methyl-r(4)-phenyl-4,5,6,7-tetrahydro-3-hydroxy-2-(benzothizol-2-yl)indazole (10): Yield 73\%; mp 177 - $179{ }^{\circ} \mathrm{C} ;{ }^{1} \mathrm{H}$ NMR $\left(400 \mathrm{MHz}, \mathrm{DMSO}-d_{6}\right) \delta 4.07$ (d, $\left.{ }^{3} J=10.6 \mathrm{~Hz}, 1 \mathrm{H}, \mathrm{H}-4 \mathrm{a}\right), 2.75$ (d, $\left.{ }^{3} J=10.64 \mathrm{~Hz}, 1 \mathrm{H}, \mathrm{H}-5 \mathrm{a}\right), 2.62$ (d, $\left.{ }^{2} J=16.84 \mathrm{~Hz}, 1 \mathrm{H}, \mathrm{H}-7 \mathrm{a}\right)$, $2.98\left(\mathrm{~d},{ }^{2} \mathrm{~J}=16.88 \mathrm{~Hz}, 1 \mathrm{H}, \mathrm{H}-7 \mathrm{e}\right), 1.27$ (s, 3H, Me at C-6), 4.86 (bs, $1 \mathrm{H}, \mathrm{OH}$ at C-6), 3.45 (s, 3H, Me of COOMe), 7.14-7.27 $(\mathrm{m}, 5 \mathrm{H}, \mathrm{Ph}-\mathrm{H}), 8.01\left(\mathrm{~d},{ }^{2} J=8.08 \mathrm{~Hz}, 1 \mathrm{H}, \mathrm{BTz}-\mathrm{Hd}\right), 7.47$ (t, $J=$ $7.68 \mathrm{~Hz}, 1 \mathrm{H}, \mathrm{BTz}-\mathrm{He}$ ), 7.32 (t, $J=7.5 \mathrm{~Hz}, 1 \mathrm{H}, \mathrm{BTz}-\mathrm{Hf}), 7.82$ (d, $\left.{ }^{2} J=8.08 \mathrm{~Hz}, 1 \mathrm{H}, \mathrm{BTz}-\mathrm{Hg}\right) ;{ }^{13} \mathrm{C}$ NMR $\left(100.6 \mathrm{MHz}, \mathrm{DMSO}-d_{6}\right)$ $\delta 152.54$ (C-3), 38.34 (C-4), 59.0 (C-5), 69.35 (C-6), 36.91 (C-7), 152.47 (C-8), 101.55 (C-9), 27.95 (Me at C-6), 51.02 (Me of COOMe), $171.93(\mathrm{C}=\mathrm{O}), 141.47$ (ipso $\mathrm{Ph}-\mathrm{C}$ ), 127.0, 127.8, 128.3 (Phenyl), 160.19 (BTz-C-b), 122.14 (BTz-C-d), 126.43 (BTz-C-e), 123.87 (BTz-C-f), 120.48 (BTz-C-g), 148.28 (BTz-C-i), 131.66 (BTz-C-h); Anal. Calcd for $\mathrm{C}_{23} \mathrm{H}_{21} \mathrm{~N}_{3} \mathrm{O}_{4} \mathrm{~S}$ (435.5): C 63.43, H 4.86, N 9.65. Found: C 63.31, H 4.75, N 9.48 . 
Synthesis of $t(5)$-methoxycarbonyl-t(6)-hydroxy-c(6)-methyl-r(4)-(p-fluorophenyl)-4,5,6,7-tetrahydro-3-hydroxy-2(benzothizol-2-yl)indazole (11): Yield 77\%; mp 217 - $220^{\circ} \mathrm{C}$; ${ }^{1} \mathrm{H}$ NMR $\left(400 \mathrm{MHz}, \mathrm{DMSO}-d_{6}\right) \delta 4.10\left(\mathrm{~d},{ }^{3} J=10.6 \mathrm{~Hz}, 1 \mathrm{H}\right.$, H-4a), 2.76 (d, $\left.{ }^{3} J=10.6 \mathrm{~Hz}, 1 \mathrm{H}, \mathrm{H}-5 \mathrm{a}\right), 2.64\left(\mathrm{~d},{ }^{2} J=17.2 \mathrm{~Hz}\right.$, $1 \mathrm{H}, \mathrm{H}-7 \mathrm{a}), 2.99$ (d, $\left.{ }^{2} J=16.88 \mathrm{~Hz}, 1 \mathrm{H}, \mathrm{H}-7 \mathrm{e}\right), 1.28$ (s, 3H, Me at C-6), 4.89 (bs, $1 \mathrm{H}, \mathrm{OH}$ at C-6), 3.46 (s, 3H, Me of COOMe), 7.20, 7.07 (4H, Ph-H), 8.0 (d, $\left.{ }^{2} J=7.68 \mathrm{~Hz}, 1 \mathrm{H}, \mathrm{BTz}-\mathrm{Hd}\right), 7.46$ (t, $J=7.52 \mathrm{~Hz}, 1 \mathrm{H}, \mathrm{BTz}-\mathrm{He}), 7.31$ (t, $J=7.52 \mathrm{~Hz}, 1 \mathrm{H}, \mathrm{BTz}-\mathrm{Hf})$, $7.82\left(\mathrm{~d},{ }^{2} J=8.04 \mathrm{~Hz}, 1 \mathrm{H}, \mathrm{BTz}-\mathrm{Hg}\right) ;{ }^{13} \mathrm{C} \mathrm{NMR}(100.6 \mathrm{MHz}$, DMSO-d $\left.d_{6}\right) \delta 152.57$ (C-3), 37.67 (C-4), 58.91(C-5), 69.35 (C-6), 36.96 (C-7), 152.38 (C-8), 101.36 (C-9), 27.93 (Me at C-6), 51.05 (Me of COOMe), $171.88(\mathrm{C}=\mathrm{O}), 137.49,114.87$, 114.66, 129.98, 129.9, 162.16, 159.76 (Phenyl), 160.1 (BTzC-b), 122.12 (BTz-C-d), 126.40 (BTz-C-e), 123.87 (BTz-C-f), 120.50 (BTz-C-g), 148.3 (BTz-C-i), 131.69 (BTz-C-h); Anal. Calcd for $\mathrm{C}_{23} \mathrm{H}_{20} \mathrm{FN}_{3} \mathrm{O}_{4} \mathrm{~S}$ (453.49): C 60.92, H 4.45, N 9.27. Found: C 60.80, H 4.31, N 9.11.

Synthesis of $t(5)$-methoxycarbonyl-t(6)-hydroxy-c(6)-methyl-r(4)-( $p$-chlorophenyl)-4,5,6,7-tetrahydro-3-hydroxy-2(benzothizol-2-yl)indazole (12): Yield 62\%; mp $269-271^{\circ} \mathrm{C}$; ${ }^{1} \mathrm{H}$ NMR $\left(400 \mathrm{MHz}, \mathrm{DMSO}-d_{6}\right) \delta 4.08\left(\mathrm{~d},{ }^{3} J=10.6 \mathrm{~Hz}, 1 \mathrm{H}\right.$, H-4a), 2.76 (d, ${ }^{3} J=10.64$ Hz, 1H, H-5a), 2.62 (d, ${ }^{2} J=17.24$ $\mathrm{Hz}, 1 \mathrm{H}, \mathrm{H}-7 \mathrm{a}), 2.99$ (d, ${ }^{2} J=17.24 \mathrm{~Hz}, 1 \mathrm{H}, \mathrm{H}-7 \mathrm{e}$ ), 1.28 (s, 3H, Me at C-6), 4.91 (bs, 1H, OH at C-6), 3.47 (s, 3H, Me of COOMe), 7.19, 7.30-7.34 (4H, Ph-H), 8.01 (d, ${ }^{2} J=8.04$ Hz, 1H, BTz$\mathrm{Hd}), 7.47(\mathrm{t}, J=7.7 \mathrm{~Hz}, 1 \mathrm{H}, \mathrm{BTz}-\mathrm{He}), 7.32(\mathrm{t}, J=7.72 \mathrm{~Hz}, 1 \mathrm{H}$, BTz-Hf), $7.82\left(\mathrm{~d},{ }^{2} J=8.04 \mathrm{~Hz}, 1 \mathrm{H}, \mathrm{BTz}-\mathrm{Hg}\right) ;{ }^{13} \mathrm{C}$ NMR $(100.6$ $\left.\mathrm{MHz}, \mathrm{DMSO}-d_{6}\right) \delta 152.48$ (C-3), 37.76 (C-4), 58.63 (C-5), 69.33 (C-6), 36.91 (C-7), 152.37 (C-8), 101.08 (C-9), 27.9 (Me at C-6), 51.11 (Me of COOMe), $171.75(\mathrm{C}=\mathrm{O}), 130.97,128.04$, 130.04, 140.49 (Phenyl), 160.05 (BTz-C-b), 122.14 (BTz-C-d), 126.42 (BTz-C-e), 123.89 (BTz-C-f), 120.49 (BTz-C-g), 148.24 (BTz-C-i), 131.67 (BTz-C-h); Anal. Calcd for $\mathrm{C}_{23} \mathrm{H}_{20} \mathrm{ClN}_{3} \mathrm{O}_{4} \mathrm{~S}$ (469.94): C 58.78, H 4.29, N 8.94. Found: C 58.67, H 4.14, N 8.81 .

Synthesis of $t(5)$-methoxycarbonyl-t(6)-hydroxy-c(6)-methyl-r(4)-(p-methoxyphenyl)-4,5,6,7-tetrahydro-3-hydroxy2-(benzothizol-2-yl)indazole (13): Yield 74\%; mp 233 - $234{ }^{\circ} \mathrm{C}$; ${ }^{1} \mathrm{H}$ NMR (400 MHz, DMSO- $\left.d_{6}\right) \delta 4.03\left(\mathrm{~d},{ }^{3} J=10.36 \mathrm{~Hz}, 1 \mathrm{H}\right.$, H-4a), 2.72 (d, $\left.{ }^{3} J=10.76 \mathrm{~Hz}, 1 \mathrm{H}, \mathrm{H}-5 \mathrm{a}\right), 2.62\left(\mathrm{~d},{ }^{2} J=17.04\right.$ $\mathrm{Hz}, 1 \mathrm{H}, \mathrm{H}-7 \mathrm{a}$ ), 2.97 (d, ${ }^{2} \mathrm{~J}=17.0 \mathrm{~Hz}, 1 \mathrm{H}, \mathrm{H}-7 \mathrm{e}$ ), 1.27 (s, 3H, Me at C-6), 4.83 (bs, $1 \mathrm{H}, \mathrm{OH}$ at C-6), 3.46 (s, 3H, Me of COOMe), $3.71\left(\mathrm{~s}, 3 \mathrm{H}, \mathrm{Me}\right.$ of $\left.\mathrm{Ph}-\mathrm{OCH}_{3}\right), 6.81,7.07$ (4H, Ph-H), 8.01(d, $\left.{ }^{2} J=7.48 \mathrm{~Hz}, 1 \mathrm{H}, \mathrm{BTz}-\mathrm{Hd}\right), 7.46$ (t, $\left.J=7.68 \mathrm{~Hz}, 1 \mathrm{H}, \mathrm{BTz}-\mathrm{He}\right)$, 7.32 (t, $J=7.48 \mathrm{~Hz}, 1 \mathrm{H}, \mathrm{BTz}-\mathrm{Hf}), 7.82\left(\mathrm{~d},{ }^{2} J=8.28 \mathrm{~Hz}, 1 \mathrm{H}\right.$, BTz-Hg); ${ }^{13} \mathrm{C}$ NMR (100.6 MHz, DMSO- $\left.d_{6}\right) \delta 152.55$ (C-3), 37.49 (C-4), 59.11(C-5), 69.36 (C-6), 36.94 (C-7), 152.37 (C-8), 101.90 (C-9), 27.99 (Me at C-6), 51.02 (Me of COOMe), 172.04 $(\mathrm{C}=\mathrm{O}), 133.23,113.43,129.05,157.77$ (Phenyl), 160.24 (BTzC-b), 122.12 (BTz-C-d), 126.40 (BTz-C-e), 123.85 (BTz-C-f), 120.47 (BTz-C-g), 148.28 (BTz-C-i), 131.67 (BTz-C-h); Anal. Calcd for $\mathrm{C}_{24} \mathrm{H}_{23} \mathrm{~N}_{3} \mathrm{O}_{5} \mathrm{~S}$ (465.52): C 61.92, H 4.98, N 9.03. Found: C 61.78, H 4.82, N 8.91.

Synthesis of $t(5)$-methoxycarbonyl-t(6)-hydroxy-c(6)-methyl-r(4)-(m-phenoxy-phenyl)-4,5,6,7-tetrahydro-3-hydroxy2-(benzothizol-2-yl)indazole(14): Yield 65\%; mp 249 - $252{ }^{\circ} \mathrm{C}$; ${ }^{1} \mathrm{H}$ NMR (400 MHz, DMSO- $\left.d_{6}\right) \delta 4.05\left(\mathrm{~d},{ }^{3} J=10.8 \mathrm{~Hz}, 1 \mathrm{H}\right.$,
H-4a), 2.75 (d, $\left.{ }^{3} J=10.8 \mathrm{~Hz}, 1 \mathrm{H}, \mathrm{H}-5 \mathrm{a}\right), 2.59\left(\mathrm{~d},{ }^{2} J=17.04 \mathrm{~Hz}\right.$, $1 \mathrm{H}, \mathrm{H}-7 \mathrm{a}$ ), 2.96 (d, ${ }^{2} \mathrm{~J}=16.6 \mathrm{~Hz}, 1 \mathrm{H}, \mathrm{H}-7 \mathrm{e}$ ), 1.26 (s, 3H, Me at C-6), 4.86 (bs, $1 \mathrm{H}, \mathrm{OH}$ at C-6), 3.48 (s, 3H, Me of COOMe), 6.86, 6.95, 7.04, 7.30, (9H, Ph-H), 8.04 (d, ${ }^{2} J=7.92 \mathrm{~Hz}, 1 \mathrm{H}$, BTz-Hd), 7.48 (t, $J=7.68 \mathrm{~Hz}, 1 \mathrm{H}, \mathrm{BTz}-\mathrm{He}), 7.34$ (t, $J=7.48$ $\mathrm{Hz}, 1 \mathrm{H}, \mathrm{BTz}-\mathrm{Hf}), 7.82$ (d, $\left.{ }^{2} J=8.32 \mathrm{~Hz}, 1 \mathrm{H}, \mathrm{BTz}-\mathrm{Hg}\right) ;{ }^{13} \mathrm{C} \mathrm{NMR}$ $\left(100.6 \mathrm{MHz}, \mathrm{DMSO}-d_{6}\right) \delta 152.65(\mathrm{C}-3), 38.40(\mathrm{C}-4), 58.97$ (C-5), 69.5 (C-6), 37.02 (C-7), 152.65 (C-8), 101.39 (C-9), 28.03 (Me at C-6), 51.30 (Me of COOMe), $172.1(\mathrm{C}=\mathrm{O}), 117.48$, $117.96,119.12,123.13,129.96,130.02,143.83,155.91,157.22$ (Phenyl), 160.29 (BTz-b), 122.32 (BTz-C-d), 126.66 (BTz-C-e), 123.86 (BTz-C-f), 120.73 (BTz-C-g), 148.42 (BTz-C-i), 131.82 (BTz-C-h); Anal. Calcd for $\mathrm{C}_{29} \mathrm{H}_{25} \mathrm{~N}_{3} \mathrm{O}_{5} \mathrm{~S}$ (527.59): C 66.02, H 4.78, N 7.96. Found: C 65.89, H 4.61, N 7.81.

Synthesis of $t(5)$-methoxycarbonyl-t(6)-hydroxy-c(6)-methyl-r(4)-(3,5-dimethoxyphenyl)-4,5,6,7-tetrahydro-3-hydroxy2-(benzothizol-2-yl)indazole (15): Yield 67\%; mp 226 - $232{ }^{\circ} \mathrm{C}$; ${ }^{1} \mathrm{H}$ NMR (400 MHz, DMSO- $\left.d_{6}\right) \delta 4.0\left(\mathrm{~d},{ }^{3} J=10.36 \mathrm{~Hz}, 1 \mathrm{H}\right.$, H-4a), $2.76\left(\mathrm{~d},{ }^{3} J=10.36 \mathrm{~Hz}, 1 \mathrm{H}, \mathrm{H}-5 \mathrm{a}\right), 2.59\left(\mathrm{~d},{ }^{2} J=17.0 \mathrm{~Hz}\right.$, 1H, H-7a), 2.98 (d, ${ }^{2} J=17.04$ Hz, 1H, H-7e), 1.26 (s, 3H, Me at C-6), 4.83 (bs, 1H, OH at C-6), 3.50 (s, 3H, Me of COOMe), 3.68 (s, 6H, 2Me of $\left.\mathrm{Ph}_{-} \mathrm{OCH}_{3}\right), 6.29-6.39$ (5H, Ph-H), 8.02 (d, $\left.{ }^{2} J=7.88 \mathrm{~Hz}, 1 \mathrm{H}, \mathrm{BTz}-\mathrm{Hd}\right), 7.47$ (t, $\left.J=7.68 \mathrm{~Hz}, 1 \mathrm{H}, \mathrm{BTz}-\mathrm{He}\right)$, 7.33 (t, $J=7.48 \mathrm{~Hz}, 1 \mathrm{H}, \mathrm{BTz}-\mathrm{Hf}), 7.82\left(\mathrm{~d},{ }^{2} J=8.32 \mathrm{~Hz}, 1 \mathrm{H}\right.$, BTz-Hg); ${ }^{13} \mathrm{C}$ NMR (100.6 MHz, DMSO- $\left.d_{6}\right) \delta 152.57$ (C-3), 38.54 (C-4), 58.73 (C-5), 69.37 (C-6), 36.87 (C-7), 152.43 (C-8), 101.37 (C-9), 27.94 (Me at C-6), 51.11 (Me of COOMe), 172.0 $(\mathrm{C}=\mathrm{O}), 55.01\left(2 \mathrm{OCH}_{3}\right.$ at $\left.\mathrm{Ph}\right), 144.02,160.09,106.39,97.8$ (Phenyl), 160.37 (BTz-C-b), 122.15 (BTz-C-d), 126.43 (BTzC-e), 123.89 (BTz-C-f), 120.48 (BTz-C-g), 148.28 (BTz-C-i), 131.67 (BTz-C-h); Anal. Calcd for $\mathrm{C}_{25} \mathrm{H}_{25} \mathrm{~N}_{3} \mathrm{O}_{6} \mathrm{~S}$ (495.55): C 60.59, H 5.08, N 8.48. Found: C 60.41, H 4.92, N 8.29.

Synthesis of $t(5)$-ethoxycarbonyl-t(6)-hydroxy-c(6)-methylr(4)-phenyl-4,5,6,7-tetrahydro-3-hydroxy-2-(benzothizol-2yl)indazole (16): Yield 74\%; mp $244-245{ }^{\circ} \mathrm{C}$; ${ }^{1} \mathrm{H}$ NMR (400 MHz, DMSO-d $\left.d_{6}\right) \delta .06\left(\mathrm{~d},{ }^{3} J=10.64 \mathrm{~Hz}, 1 \mathrm{H}, \mathrm{H}-4 \mathrm{a}\right), 2.72$ (d, $\left.{ }^{3} J=10.64 \mathrm{~Hz}, 1 \mathrm{H}, \mathrm{H}-5 \mathrm{a}\right), 2.61$ (d, $\left.{ }^{2} J=17.24 \mathrm{~Hz}, 1 \mathrm{H}, \mathrm{H}-7 \mathrm{a}\right)$, $2.98\left(\mathrm{~d},{ }^{2} J=16.84 \mathrm{~Hz}, 1 \mathrm{H}, \mathrm{H}-7 \mathrm{e}\right), 1.29$ (s, 3H, Me at C-6), 4.81 (bs, 1H, OH at C-6), 3.92 (m, 2H, $\mathrm{CH}_{2}$ of COOEt), 0.97 (t, 3H, Me of COOEt), 7.16-7.20, 7.23-7.27 (5H, Ph-H), 8.01 (d, ${ }^{2} J=$ $8.04 \mathrm{~Hz}, 1 \mathrm{H}, \mathrm{BTz}-\mathrm{Hd}), 7.47$ (t, $J=7.7 \mathrm{~Hz}, 1 \mathrm{H}, \mathrm{BTz}-\mathrm{He}), 7.32$ (t, $J=7.7 \mathrm{~Hz}, 1 \mathrm{H}, \mathrm{BTz}-\mathrm{Hf}), 7.82\left(\mathrm{~d},{ }^{2} J=8.08 \mathrm{~Hz}, 1 \mathrm{H}, \mathrm{BTz}-\mathrm{Hg}\right.$ ); ${ }^{13} \mathrm{C}$ NMR (100.6 MHz, DMSO-d 6 ) $\delta 152.54$ (C-3), 38.44 (C-4), 59.55 (C-5), 69.34 (C-6), 36.92 (C-7), 152.46 (C-8), 101.6 (C-9), 27.83 (Me at C-6), $58.77\left(\mathrm{CH}_{2}\right.$ of COOEt), $14.0\left(\mathrm{CH}_{3}\right.$ of COOEt), $171.48(\mathrm{C}=\mathrm{O}), 141.39$ (ipso Ar-C), 128.22, 127.95 (Phenyl), 160.19 (BTz-C-b), 122.13 (BTz-C-d), 126.4 (BTzC-e), 123.85 (BTz-C-f), 120.47 (BTz-C-g), 148.27 (BTz-C-i), 131.66 (BTz-C-h); Anal. Calcd for $\mathrm{C}_{24} \mathrm{H}_{23} \mathrm{~N}_{3} \mathrm{O}_{4} \mathrm{~S}$ (449.52): C 64.13, H 5.16, N 9.35. Found: C 64.02, H 5.04, N 9.16.

Synthesis of $t(5)$-methoxycarbonyl-t(6)-hydroxy-c(6)-methyl-r(4)-(m-chlorophenyl)-4,5,6,7-tetrahydro-3-hydroxy-2(benzothizol-2-yl)indazole (17): Yield 64\%; mp 289 - $292{ }^{\circ} \mathrm{C}$; ${ }^{1} \mathrm{H}$ NMR $\left(400 \mathrm{MHz}, \mathrm{DMSO}-d_{6}\right) \delta 4.07\left(\mathrm{~d},{ }^{3} J=10.24 \mathrm{~Hz}, 1 \mathrm{H}\right.$, H-4a), 2.82 (d, $\left.{ }^{3} J=10.64 \mathrm{~Hz}, 1 \mathrm{H}, \mathrm{H}-5 \mathrm{a}\right), 2.55-2.63$ (H-7a), $3.01\left(\mathrm{~d},{ }^{2} \mathrm{~J}=16.88 \mathrm{~Hz}, 1 \mathrm{H}, \mathrm{H}-7 \mathrm{e}\right), 1.28$ (s, 3H, Me at C-6), 4.92 (bs, 1H, OH at C-6), 3.47 (s, 3H, Me of COOMe), 7.04-7.30 $(4 \mathrm{H}, \mathrm{Ph}-\mathrm{H}), 8.01\left(\mathrm{~d},{ }^{2} J=7.72 \mathrm{~Hz}, 1 \mathrm{H}, \mathrm{BTz}-\mathrm{Hd}\right), 7.47$ (t, $J=$ 
$7.8 \mathrm{~Hz}, 1 \mathrm{H}, \mathrm{BTz}-\mathrm{He}), 7.33$ (t, $J=7.68 \mathrm{~Hz}, 1 \mathrm{H}, \mathrm{BTz}-\mathrm{Hf}), 7.82$ (d, $\left.{ }^{2} J=8.08 \mathrm{~Hz}, 1 \mathrm{H}, \mathrm{BTz}-\mathrm{Hg}\right) ;{ }^{13} \mathrm{C}$ NMR $\left(100.6 \mathrm{MHz}, \mathrm{DMSO}-d_{6}\right)$ $\delta 152.52$ (C-3), 38.16 (C-4), 58.4(C-5), 69.38 (C-6), 36.9 (C-7), 152.43 (C-8), 100.82 (C-9), 27.91 (Me at C-6), 51.11 (Me of COOMe), $171.74(\mathrm{C}=\mathrm{O}), 144.1,132.68,129.91,127.86,127.18$, 126.6 (Phenyl), 160.02 (BTz-b), 122.16 (BTz-C-d), 126.45 (BTz-C-e), 123.93 (BTz-C-f), 120.52 (BTz-C-g), 148.25 (BTzC-i), 131.67 (BTz-C-h); Anal. Calcd for $\mathrm{C}_{23} \mathrm{H}_{20} \mathrm{ClN}_{3} \mathrm{O}_{4} \mathrm{~S}$ (469.94): C 58.78, H 4.29, N 8.94. Found: C 58.61, H 4.13, N 8.81.

Synthesis of $t(5)$-ethoxycarbonyl-t(6)-hydroxy-c(6)-methylr(4)-( $p$-nitrophenyl)-4,5,6,7-tetrahydro-3-hydroxy-2-(benzothizol-2-yl)indazole (18): Yield 52\%; mp $260-262{ }^{\circ} \mathrm{C} ;{ }^{1} \mathrm{H}$ NMR (400 MHz, DMSO- $\left.d_{6}\right) \delta 4.23\left(\mathrm{~d},{ }^{3} J=9.92 \mathrm{~Hz}, 1 \mathrm{H}, \mathrm{H}-4 \mathrm{a}\right)$, 2.80-2.83 (H-5a), 2.62 (H-7a), 3.03 (d, $\left.{ }^{2} J=17.2 \mathrm{~Hz}, 1 \mathrm{H}, \mathrm{H}-7 \mathrm{e}\right)$, 1.32 (s, 3H, Me at C-6), 4.98 (bs, $1 \mathrm{H}, \mathrm{OH}$ at C-6), $3.92(\mathrm{~m}, 2 \mathrm{H}$, $\mathrm{CH}_{2}$ of COOEt), 0.96 (t, 3H, Me of COOEt), 7.37-7.49, 8.10$8.16(4 \mathrm{H}, \mathrm{Ph}-\mathrm{H}), 8.0$ (d, $\left.{ }^{2} J=7.68 \mathrm{~Hz}, 1 \mathrm{H}, \mathrm{BTz}-\mathrm{Hd}\right), 7.47$ (t, $J=$ $8.44 \mathrm{~Hz}, 1 \mathrm{H}, \mathrm{BTz}-\mathrm{He}), 7.32$ (t, $J=7.68 \mathrm{~Hz}, 1 \mathrm{H}, \mathrm{BTz}-\mathrm{Hf}), 7.82$ $\left(\mathrm{d},{ }^{2} J=8.04 \mathrm{~Hz}, 1 \mathrm{H}, \mathrm{BTz}-\mathrm{Hg}\right) ;{ }^{13} \mathrm{C}$ NMR $(100.6 \mathrm{MHz}, \mathrm{DMSO}-$ $\left.d_{6}\right) \delta 152.48$ (C-3), 38.40 (C-4), 58.65 (C-5), 69.4 (C-6), 36.97 (C-7), 152.36 (C-8), 100.51 (C-9), 27.81 (Me at C-6), $57.9\left(\mathrm{CH}_{2}\right.$ of COOEt), $14.03\left(\mathrm{CH}_{3}\right.$ of COOEt), $171.02(\mathrm{C}=\mathrm{O}), 151.55$, 146.35, 129.81, 123.26 (Phenyl), 159.93 (BTz-C-b), 122.18 (BTz-C-d), 126.47 (BTz-C-e), 123.97 (BTz-C-f), 120.56 (BTzC-g), 148.25 (BTz-C-i), 131.7 (BTz-C-h); Anal. Calcd for $\mathrm{C}_{24}$ $\mathrm{H}_{22} \mathrm{~N}_{4} \mathrm{O}_{6} \mathrm{~S}$ (494.52): C 58.29, H 4.48, N 11.33. Found: C 58.13, $\mathrm{H} 4.24, \mathrm{~N} 11.11$.

$\mathrm{OH}$ proton of compounds 10-18 at C-3 position observed as a broad singlet with the integral values of $(\sim 0.3-0.6)$ at around 10 - 11 ppm.

General procedure for the synthesis $r(4)$-aryl-t(5)-alkoxycarbonyl-t(6)-hydroxy-c(6)-methyl-4,5,6,7-tetrahydro-3-hydroxy-1(H)indazoles (19-27). A mixture of cyclic ketoester (1 $\mathrm{mmol})$ and methyl hydrazino carboxylate $(1.2 \mathrm{mmol})$ in toluene with the addition of catalytic amount of acetic acid were refluxed for about 2 - 4 hrs. After completion of the reaction, the solvent was evaporated under vacuum and the resultant residue was recrystalized from ethanol.

Synthesis of $t(5)$-methoxycarbonyl-t(6)-hydroxy-c(6)-methyl-r(4)-phenyl-4,5,6,7-tetrahydro-3-hydroxy-1(H)-indazole (19): Yield 89\%; mp $295-296{ }^{\circ} \mathrm{C} ;{ }^{1} \mathrm{H}$ NMR (400 MHz, DMSO$\left.d_{6}\right) \delta 4.05\left(\mathrm{~d},{ }^{3} J=10.6 \mathrm{~Hz}, 1 \mathrm{H}, \mathrm{H}-4 \mathrm{a}\right), 2.61\left(\mathrm{~d},{ }^{3} J=10.6 \mathrm{~Hz}\right.$, $1 \mathrm{H}, \mathrm{H}-5 \mathrm{a}), 2.54$ (d, $\left.{ }^{2} \mathrm{~J}=16.48 \mathrm{~Hz}, 1 \mathrm{H}, \mathrm{H}-7 \mathrm{a}\right), 2.77$ (d, ${ }^{2} \mathrm{~J}=$ $15.76 \mathrm{~Hz}, 1 \mathrm{H}, \mathrm{H}-7 \mathrm{e}), 1.20$ (s, 3H, Me at C-6), 4.51 (bs, $1 \mathrm{H}, \mathrm{OH}$ at C-6), 3.42 (s, 3H, Me of COOMe), 7.07, 7.14, $7.20(5 \mathrm{H}$, $\mathrm{Ph}-\mathrm{H}) ;{ }^{13} \mathrm{C}$ NMR (100.6 MHz, DMSO- $\left.d_{6}\right) \delta 158.01(\mathrm{C}-3), 38.72$ (C-4), 59.94 (C-5), 69.95 (C-6), 36.54 (C-7), 138.78 (C-8), 99.92 (C-9), 28.49 (Me at C-6), 50.84 (Me of COOMe), 172.65 $(\mathrm{C}=\mathrm{O}), 143.05$ (ipso Ar-C), 126.04, 127.78, 128.07 (Phenyl); Anal. Calcd for $\mathrm{C}_{16} \mathrm{H}_{18} \mathrm{~N}_{2} \mathrm{O}_{4}$ (302.13): C 63.56, H 6.0, N 9.27. Found: C 63.50, H 5.89, N 9.19.

Synthesis of $t(5)$-methoxycarbonyl-t(6)-hydroxy-c(6)-methyl-r(4)-(p-fluorophenyl)-4,5,6,7-tetrahydro-3-hydroxy-1(H)indazole (20): Yield $82 \%$; mp $240-243{ }^{\circ} \mathrm{C} ;{ }^{1} \mathrm{H}$ NMR (400 MHz, DMSO-d $\left.d_{6}\right) \delta 4.07$ (d, $\left.{ }^{3} J=10.4 \mathrm{~Hz}, 1 \mathrm{H}, \mathrm{H}-4 \mathrm{a}\right), 2.58$ (d, $\left.{ }^{3} J=10.1 \mathrm{~Hz}, 1 \mathrm{H}, \mathrm{H}-5 \mathrm{a}\right), 2.55\left(\mathrm{~d},{ }^{2} J=14.68 \mathrm{~Hz}, 1 \mathrm{H}, \mathrm{H}-7 \mathrm{a}\right)$, $2.77\left(\mathrm{~d},{ }^{2} \mathrm{~J}=16.0 \mathrm{~Hz}, 1 \mathrm{H}, \mathrm{H}-7 \mathrm{e}\right), 1.22$ (s, 3H, Me at C-6), 4.49 (bs, 1H, OH at C-6), 3.44 (s, 3H, Me of COOMe), 7.0-7.12 (4H,
$\mathrm{Ph}-\mathrm{H}) ;{ }^{13} \mathrm{C}$ NMR (100.6 MHz, DMSO- $\left.d_{6}\right) \delta 157.98$ (C-3), 38.08 (C-4), 59.9 (C-5), 69.97 (C-6), 36.56 (C-7), 138.91 (C-8), 99.28 (C-9), 28.43 (Me at C-6), 50.8 (Me of COOMe), $172.58(\mathrm{C}=\mathrm{O})$, 139.02, 114.86, 114.51, 129.82, 129.75, 161.94, 159.55 (Phenyl); Anal. Calcd for $\mathrm{C}_{16} \mathrm{H}_{17} \mathrm{FN}_{2} \mathrm{O}_{4}$ (320.32): C 59.99, H 5.35, N 8.75. Found: C 59.91, H 5.29, N 8.68.

Synthesis of $t(5)-m e t h o x y c a r b o n y l-t(6)-h y d r o x y-c(6)-m e-$ thyl-r(4)-(p-chlorophenyl)-4,5,6,7-tetrahydro-3-hydroxy-1(H)indazole (21): Yield $72 \%$; mp $315-317{ }^{\circ} \mathrm{C} ;{ }^{1} \mathrm{H}$ NMR (400 MHz, DMSO- $\left.d_{6}\right) \delta 4.05\left(\mathrm{~d},{ }^{3} J=10.28 \mathrm{~Hz}, 1 \mathrm{H}, \mathrm{H}-4 \mathrm{a}\right), 2.57$ (d, $\left.{ }^{3} J=10.6 \mathrm{~Hz}, 1 \mathrm{H}, \mathrm{H}-5 \mathrm{a}\right), 2.55$ (1H, H-7a), 2.77 (d, ${ }^{2} J=16.12$ $\mathrm{Hz}, 1 \mathrm{H}, \mathrm{H}-7 \mathrm{e}$ ), 1.20 (s, 3H, Me at C-6), 4.56 (bs, $1 \mathrm{H}, \mathrm{OH}$ at $\mathrm{C}-6), 3.44$ (s, 3H, Me of COOMe), 7.09,7.26 (4H, Ph-H); ${ }^{13} \mathrm{C}$ NMR (100.6 MHz, DMSO- $d_{6}$ ) $\delta 157.9$ (C-3), 38.15 (C-4), 59.61 (C-5), 69.94 (C-6), 36.55 (C-7), 138.76 (C-8), 98.99 (C-9), 28.42 (Me at C-6), 50.94 (Me of COOMe), $172.43(\mathrm{C}=\mathrm{O}), 127.77$, 129.99, 130.51, 142.1 (Phenyl); Anal. Calcd for $\mathrm{C}_{16} \mathrm{H}_{17} \mathrm{ClN}_{2} \mathrm{O}_{4}$ (336.77): C 57.06, H 5.09, N 8.32. Found: C 56.97, H 5.01, N 8.26 .

Synthesis of $t(5)$-methoxycarbonyl-t(6)-hydroxy-c(6)-methyl-r(4)-(p-methoxyphenyl)-4,5,6,7-tetrahydro-3-hydroxy1(H)-indazole (22): Yield 80\%; mp $250-253{ }^{\circ} \mathrm{C} ;{ }^{1} \mathrm{H}$ NMR (400 MHz, DMSO-d $) \delta 4.0\left(\mathrm{~d},{ }^{3} J=10.4 \mathrm{~Hz}, 1 \mathrm{H}, \mathrm{H}-4 \mathrm{a}\right), 2.54$ $\left(\mathrm{d},{ }^{3} J=9.96 \mathrm{~Hz}, 1 \mathrm{H}, \mathrm{H}-5 \mathrm{a}\right), 2.51$ (H-7a), $2.75\left(\mathrm{~d},{ }^{2} J=16.04 \mathrm{~Hz}\right.$, $1 \mathrm{H}, \mathrm{H}-7 \mathrm{e}), 1.20$ (s, 3H, Me at C-6), 4.48 (bs, 1H, OH at C-6), 3.44 (s, 3H, Me of COOMe), 6.77, $6.98(4 \mathrm{H}, \mathrm{Ph}-\mathrm{H}) ;{ }^{13} \mathrm{C} \mathrm{NMR}$ $\left(100.6 \mathrm{MHz}, \mathrm{DMSO}-d_{6}\right) \delta 157.56(\mathrm{C}-3), 37.89$ (C-4), 60.1 (C-5), 69.98 (C-6), 36.53 (C-7), 138.81 (C-8), 99.58 (C-9), 28.5 (Me at C-6), 50.95 (Me of COOMe), $172.78(\mathrm{C}=\mathrm{O}), 113.21$, 113.59, 127.4, 128.96, 134.82, 158.14 (Phenyl); Anal. Calcd for $\mathrm{C}_{17} \mathrm{H}_{20} \mathrm{~N}_{2} \mathrm{O}_{5}$ (332.35): C 61.44, H 6.07, N 8.43. Found: $\mathrm{C}$ $61.37, \mathrm{H} 6.01, \mathrm{~N} 8.37$.

Synthesis of $t(5)$-methoxycarbonyl-t(6)-hydroxy-c(6)-methyl-r(4)-(m-phenoxyphenyl)-4,5,6,7-tetrahydro-3-hydroxy1(H)-indazole (23): Yield 77\%; mp $263-265{ }^{\circ} \mathrm{C} ;{ }^{1} \mathrm{H}$ NMR (400 MHz, DMSO-d $) \delta 4.05$ (d, $\left.{ }^{3} J=10.24 \mathrm{~Hz}, 1 \mathrm{H}, \mathrm{H}-4 \mathrm{a}\right)$, $2.59\left(\mathrm{~d},{ }^{3} J=10.64 \mathrm{~Hz}, 1 \mathrm{H}, \mathrm{H}-5 \mathrm{a}\right), 2.51\left(\mathrm{~d},{ }^{2} J=15.76 \mathrm{~Hz}, 1 \mathrm{H}\right.$, H-7a), 2.76 (d, $\left.{ }^{2} J=15.76 \mathrm{~Hz}, 1 \mathrm{H}, \mathrm{H}-7 \mathrm{e}\right), 1.20$ (s, 3H, Me at C-6), 4.53 (bs, 1H, OH at C-6), 3.44 (s, 3H, Me of COOMe), 6.74-7.36 (9H, Ph-H); ${ }^{13} \mathrm{C}$ NMR (100.6 MHz, DMSO- $\left.d_{6}\right) \delta$ 157.23 (C-3), 38.64 (C-4), 59.71 (C-5), 69.93 (C-6), 36.49 (C-7), 138.84 (C-8), 98.92 (C-9), 28.41 (Me at C-6), 50.92 (Me of COOMe), $172.55(\mathrm{C}=\mathrm{O}), 117.04,117.71,119.09,122.84$, 123.72, 129.43, 129.89, 145.37, 155.56, 157.23 (Phenyl); Anal. Calcd for $\mathrm{C}_{22} \mathrm{H}_{22} \mathrm{~N}_{2} \mathrm{O}_{5}$ (394.42): C 66.99, H 5.62, N 7.10. Found: C 66.91, H 5.54, N 7.02.

Synthesis of $t(5)$-methoxycarbonyl-t(6)-hydroxy-c(6)-methyl-r(4)-(3,5-methoxy-phenyl)-4,5,6,7-tetrahydro-3-hydroxy1(H)-indazole (24): Yield 70\%; mp $245-247{ }^{\circ} \mathrm{C} ;{ }^{1} \mathrm{H}$ NMR (400 MHz, DMSO-d 6 ) $\delta 3.98$ (d, $\left.{ }^{3} J=10.28 \mathrm{~Hz}, 1 \mathrm{H}, \mathrm{H}-4 \mathrm{a}\right)$, 2.57 (d, $\left.{ }^{3} J=10.28 \mathrm{~Hz}, 1 \mathrm{H}, \mathrm{H}-5 \mathrm{a}\right), 2.52$ (d, ${ }^{2} J=15.04 \mathrm{~Hz}, 1 \mathrm{H}$, H-7a), 2.76 (d, ${ }^{2} J=15.76 \mathrm{~Hz}, 1 \mathrm{H}, \mathrm{H}-7 \mathrm{e}$ ), 1.19 (s, 3H, Me at C-6), 4.48 (bs, 1H, OH at C-6), 3.47 (s, 3H, Me of COOMe), 3.66 (s, 6H, 2Me of Ph-OMe), 6.20, 6.28 (3H, Ph-H); ${ }^{13} \mathrm{C} \mathrm{NMR}$ (100.6 MHz, DMSO-d6) $\delta 158.01$ (C-3), 38.93 (C-4), 59.74 (C-5), 69.96 (C-6), 36.49 (C-7), 138.85 (C-8), 97.64 (C-9), 28.47 (Me at C-6), 50.94 (Me of COOMe), $172.72(\mathrm{C}=\mathrm{O}), 54.93$ (2Me of Ph-OMe), 99.0, 106.28, 145.60, 159.89 (Phenyl); Anal. 
Calcd for $\mathrm{C}_{18} \mathrm{H}_{22} \mathrm{~N}_{2} \mathrm{O}_{6}$ (362.38): C 59.66, H 6.12, N 7.73. Found: C 59.60, H 6.07, N 7.66.

Synthesis of $t(5)$-ethoxycarbonyl-t(6)-hydroxy-c(6)-methylr(4)-phenyl-4,5,6,7-tetrahydro-3-hydroxy-1(H)-indazole (25): Yield 77\%; mp $250-252{ }^{\circ} \mathrm{C} ;{ }^{1} \mathrm{H}$ NMR (400 MHz, DMSO- $d_{6}$ ) $\delta 4.04\left(\mathrm{~d},{ }^{3} J=10.36 \mathrm{~Hz}, 1 \mathrm{H}, \mathrm{H}-4 \mathrm{a}\right), 2.55\left(\mathrm{~d},{ }^{3} J=10.4 \mathrm{~Hz}, 1 \mathrm{H}\right.$, H-5a), $2.53\left(\mathrm{~d},{ }^{2} J=16.6 \mathrm{~Hz}, 1 \mathrm{H}, \mathrm{H}-7 \mathrm{a}\right), 2.76\left(\mathrm{~d},{ }^{2} J=16.12 \mathrm{~Hz}\right.$, $1 \mathrm{H}, \mathrm{H}-7 \mathrm{e}), 1.22$ (s, 3H, Me at C-6), 4.45 (bs, 1H, OH at C-6), 3.89 (m, 2H, $\mathrm{CH}_{2}$ of COOEt), 0.95 ( $\mathrm{t}, 3 \mathrm{H}, \mathrm{Me}$ of COOEt), 7.06$7.21(5 \mathrm{H}, \mathrm{Ph}-\mathrm{H}) ;{ }^{13} \mathrm{C}$ NMR $\left(100.6 \mathrm{MHz}, \mathrm{DMSO}-d_{6}\right) \delta 158.0$ (C-3), 38.87 (C-4), 59.69 (C-5), 69.95 (C-6), 36.54 (C-7), 138.74 (C-8), 99.32 (C-9), 28.39 (Me at C-6), 59.32 ( $\mathrm{CH}_{2}$ of COOEt), 13.99 (Me of COOEt), $172.22(\mathrm{C}=\mathrm{O}), 142.95$ (ipso Ar-C), 128.18, 127.68, 125.98 (Phenyl); Anal. Calcd for $\mathrm{C}_{17} \mathrm{H}_{20} \mathrm{~N}_{2} \mathrm{O}_{4}$ (316.35): C 64.54, H 6.37, N 8.86. Found: C 64.48, H 6.31, N 8.81 .

Synthesis of $t(5)$-methoxycarbonyl-t(6)-hydroxy-c(6)-methyl-r(4)-(m-chlorophenyl)-4,5,6,7-tetrahydro-3-hydroxy1(H)-indazole (26): Yield 71\%; mp 297 - $299{ }^{\circ} \mathrm{C}$; ${ }^{1} \mathrm{H}$ NMR (400 MHz, DMSO- $\left.d_{6}\right) \delta 4.07$ (d, $\left.{ }^{3} J=10.24 \mathrm{~Hz}, 1 \mathrm{H}, \mathrm{H}-4 \mathrm{a}\right)$, $2.63\left(\mathrm{~d},{ }^{3} J=10.64 \mathrm{~Hz}, 1 \mathrm{H}, \mathrm{H}-5 \mathrm{a}\right), 2.53\left(\mathrm{~d},{ }^{2} J=16.84 \mathrm{~Hz}, 1 \mathrm{H}\right.$, H-7a), 2.8 (d, $\left.{ }^{2} J=16.12 \mathrm{~Hz}, 1 \mathrm{H}, \mathrm{H}-7 \mathrm{e}\right), 1.21$ (s, 3H, Me at C-6), 4.58 (bs, $1 \mathrm{H}, \mathrm{OH}$ at C-6), 3.44 (s, 3H, Me of COOMe), 7.06, 7.19-7.27 (4H, Ph-H); ${ }^{13} \mathrm{C}$ NMR (100.6 MHz, DMSO- $\left.d_{6}\right) \delta$ 157.93 (C-3), 38.54 (C-4), 59.46 (C-5), 69.98 (C-6), 36.51 (C-7), 138.98 (C-8), 98.8 (C-9), 28.4 (Me at C-6), 50.93 (Me of COOMe), $172.43(\mathrm{C}=\mathrm{O}), 126.18,127.06,127.78,129.66$, 132.45, 145.76 (Phenyl); Anal. Calcd for $\mathrm{C}_{16} \mathrm{H}_{17} \mathrm{ClN}_{2} \mathrm{O}_{4}$ (336.77): C 57.06, H 5.09, N 8.32. Found: C 57.01, H 5.02, N 8.27.

Synthesis of $t(5)$-ethoxycarbonyl- $t(6)-h y d r o x y-c(6)$-methylr(4)-(p-nitrophenyl)-4,5,6,7-tetrahydro-3-hydroxy-1(H)-indazole (27): Yield $70 \%$; mp $285-287{ }^{\circ} \mathrm{C} ;{ }^{1} \mathrm{H}$ NMR $(400 \mathrm{MHz}$, DMSO-d $\left.d_{6}\right) \delta .22\left(\mathrm{~d},{ }^{3} J=10.64 \mathrm{~Hz}, 1 \mathrm{H}, \mathrm{H}-4 \mathrm{a}\right), 2.80-2.83$ (d, $\left.{ }^{3} J=10.6 \mathrm{~Hz}, 1 \mathrm{H}, \mathrm{H}-5 \mathrm{a}\right), 2.56\left(\mathrm{~d},{ }^{2} J=17.2 \mathrm{~Hz}, 1 \mathrm{H}, \mathrm{H}-7 \mathrm{a}\right)$, $2.62-2.66\left(\mathrm{~d},{ }^{2} J=16.88 \mathrm{~Hz}, 1 \mathrm{H}, \mathrm{H}-7 \mathrm{e}\right), 1.26$ (s, 3H, Me at C-6), 4.63 (bs, $1 \mathrm{H}, \mathrm{OH}$ at C-6), 3.92 (m, 2H, $\mathrm{CH}_{2}$ of COOEt), 0.99 (t, 3H, Me of COOEt), 7.37-7.49, 8.10-8.16 $(4 \mathrm{H}, \mathrm{Ph}-\mathrm{H}) ;{ }^{13} \mathrm{C}$ NMR (100.6 MHz, DMSO- $\left.d_{6}\right) \delta 157.89$ (C-3), 38.88 (C-4), 59.87 (C-5), 70.0 (C-6), 36.63 (C-7), 138.99 (C-8), 98.65 (C-9), 28.31 (Me at C-6), 58.89 ( $\mathrm{CH}_{2}$ of COOEt), 14.03 (Me of COOEt), $171.7(\mathrm{C}=\mathrm{O}), 149.82,146.09,129.71,123.04$ (Phenyl); Anal. Calcd for $\mathrm{C}_{17} \mathrm{H}_{19} \mathrm{~N}_{3} \mathrm{O}_{6}$ (361.35): C 56.51, H 5.30, N 11.63. Found: C 56.43, H 5.25, N 11.57.

$\mathrm{N}-\mathrm{H}(1 \mathrm{H})$ and $\mathrm{OH}$ (at $\mathrm{C}-3$ ) protons are observed as a broad singlet in the range of $9-10 \mathrm{ppm}$ and $11-12$ ppm respectively.

Synthesis of methyl carboxylate hydrazone of r(2),c(4)-bis (methoxycarbonyl)-c(5)-hydroxy-t(5)-methyl $t(3)$-phenylcyclohexanoe (28). A mixture of cyclic ketoester 1 (1 mmol) and methyl hydrazino carboxylate $(1.1 \mathrm{mmol})$ in ethanol was refluxed for about $2 \mathrm{hrs}$. After completion of the reaction, the reaction mixture was poured in ice water and the white solid mass was filtered and recrystalized from ethanol. Yield $69 \% ;{ }^{1} \mathrm{H}$ NMR $\left(400 \mathrm{MHz}, \mathrm{CDCl}_{3}+\mathrm{DMSO}-d_{6}\right) \delta 2.65\left(\mathrm{~d},{ }^{3} J=12.04 \mathrm{~Hz}, 1 \mathrm{H}\right.$, H-2a), 3.64 (t, $\left.{ }^{3} J=12.24 \mathrm{~Hz}, 1 \mathrm{H}, \mathrm{H}-3 \mathrm{a}\right), 3.38$ (d, ${ }^{3} J=12.04$ $\mathrm{Hz}, 1 \mathrm{H}, \mathrm{H}-4 \mathrm{a}), 1.84$ (d, $\left.{ }^{2} J=14.52 \mathrm{~Hz}, 1 \mathrm{H}, \mathrm{H}-6 \mathrm{a}\right), 3.03$ (d, ${ }^{2} J=$ $14.56 \mathrm{~Hz}, 1 \mathrm{H}, \mathrm{H}-6 \mathrm{e}), 1.11$ (s, 3H, Me at C-6), $3.90(1 \mathrm{H}, \mathrm{OH}$ at C-6), 3.51 (s, 3H, Me of NNHCOOMe), 3.13, 3.32 (2s, 6H, Me of COOMe at C-2 \& C-4), 6.99-7.26 (5H, Ph-H); ${ }^{13} \mathrm{C}$ NMR $\left(100.6 \mathrm{MHz}, \mathrm{CDCl}_{3}+\mathrm{DMSO}-d_{6}\right) \delta 167.6(\mathrm{C}-1 / \mathrm{C}=\mathrm{N}), 55.8$ (C-2), 44.3 (C-3), 57.3 (C-4), 70.8 (C-5), 38.8 (C-6), 28.3 (Me at C-6), 50.9, 51.1 (Me of COOMe at C-2 \& C-4), 50.9 (Me of NNHCOOMe), 172.1, $169.3(\mathrm{C}=\mathrm{O}$ of COOMe at $\mathrm{C}-2$ \& $\mathrm{C}-4)$, $169.3(\mathrm{C}=\mathrm{O}$ of NNHCOOMe), 139.1, 127.8, 127.5, 126.6 (Phenyl).

General procedure for the synthesis $r(4)$-aryl-t(5)-alkoxycarbonyl-t(6)-hydroxy-c(6)-methyl-4,5,6,7-tetrahydro-3-hydroxy-2-(pyridin-2-yl)-indazoles (29-40). Compounds 29-40 have been synthesized by adopting the literature method. ${ }^{1}$

Antimicrobial activity. Antimicrobial activity of the synthesized compounds was tested by cup or well method. ${ }^{16}$ Solutions of test compounds were prepared in DMSO at a concentration of $125 \mu \mathrm{g} / \mathrm{mL}$. Whatman No. 1 discs (about $6 \mathrm{~mm}$ in diameter) were impregnated in the test compounds for about one hour prior to test (paper disc impregnated in DMSO only served as negative control). Commercially available drug disc (10 $\mu \mathrm{g} /$ disc $)$ was used as positive reference standard. The discs were placed on the inoculated agar plates and incubated at $37 \pm 1{ }^{\circ} \mathrm{C}$ for bacteria and $28 \pm 1{ }^{\circ} \mathrm{C}$ for fungi. Antimicrobial activity was evaluated by measuring the growth inhibition zone ( $\mathrm{mm}$ ) against the tested organisms after 24 hours for bacteria and 72 hours for fungus.

\section{Results and Discussion}

In our current research, we have focused on the synthesis of various $N$-substituted tetrahydroindazoles by using the characteristic reaction behavior of cyclic $\beta$ keto esters towards the monosubstituted hydrazine and optimization of a versatile reaction conditions to acquire as single isomer in high yield. Previously, we have reported a series of $N(2)$-pyridyl tetrahydroindazoles. ${ }^{1}$ Here, we report the synthesis and complete characterization of $N(2)$-benzothiazolyl tetrahydroindazoles, besides; activity of both $N(2)$-pyridyl and of $N(2)$-benzothiazolyl tetrahydroindazoles against some selected bacteria and fungus have been carried out.

The parent cyclic keto esters, which are categorized in the general name, 2,4-bis(alkoxycarbonyl)-3-aryl-5-hydroxy-5-methylcyclohexanones have been synthesized by the Knoevenagel condensation followed by the cyclization of various aromatic aldehydes with acetoacetic esters in presence of methylamine solution.

To accomplish the $N$-substituted tetrahydroindazoles the synthesized cyclic ketoesters have been treated with the hydrazine reagents like 2-hydrazinobenzothiazole and methylhydrazino carboxylate. In view of the better biological activities associated with benzothiazole moiety and $N$-carbomethoxy derivatives, we have attempted to synthesize a series of $N$-benzothiazolyl and $N$-carbomethoxy tetrahydroindazoles.

A series of 4-aryl-5-alkoxycarbonyl-6-hydroxy-6-methyl4,5,6,7-tetrahydro-3-hydroxy-2-(benzothiazol-2-yl)-indazoles (10-18) were synthesized by the reaction of 2-hydrazinobenzothiazole with the respective cyclic $\beta$ keto esters. The optimum reaction medium was found to be toluene/cat.qty of AcOH by performing the reaction at several conditions and also from our previous research experience. It has been found that, excess of acid resulted in the mixture of products in some cases $(\mathbf{1 7}, \mathbf{2 6}$ 
Scheme 1. Synthesis of tetrahydroindazoles 10-27

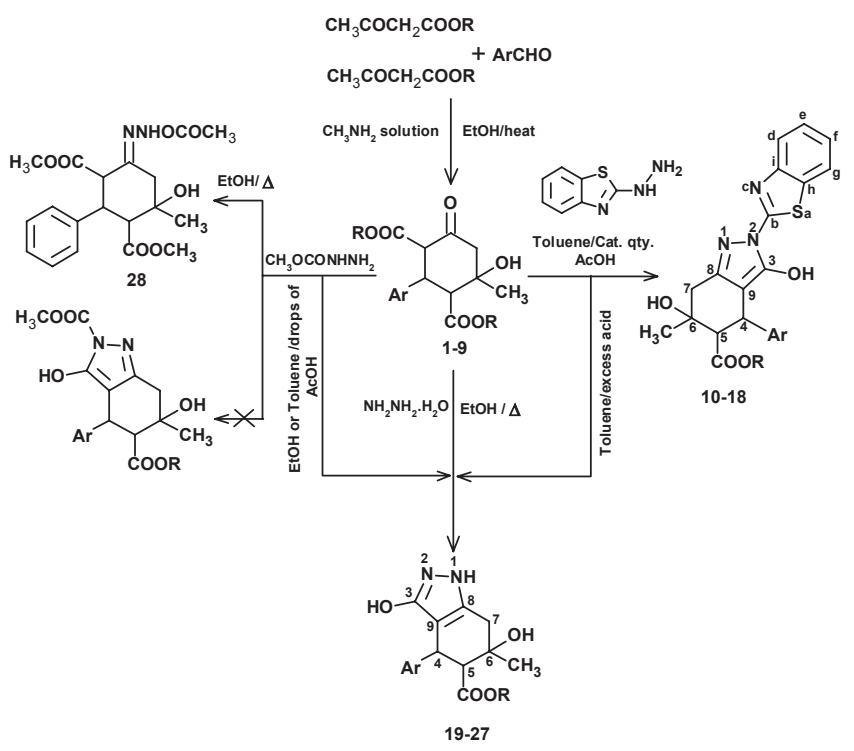

\begin{tabular}{ccccc}
\hline & Entry & & $\mathrm{R}$ & $\mathrm{Ar}$ \\
\hline $\mathbf{1}$ & $\mathbf{1 0}$ & $\mathbf{1 9}$ & $\mathrm{CH}_{3}$ & $\mathrm{Ph}$ \\
$\mathbf{2}$ & $\mathbf{1 1}$ & $\mathbf{2 0}$ & $\mathrm{CH}_{3}$ & $p-\mathrm{F}-\mathrm{Ph}$ \\
$\mathbf{3}$ & $\mathbf{1 2}$ & $\mathbf{2 1}$ & $\mathrm{CH}_{3}$ & $p-\mathrm{Cl}-\mathrm{Ph}$ \\
$\mathbf{4}$ & $\mathbf{1 3}$ & $\mathbf{2 2}$ & $\mathrm{CH}_{3}$ & $p-\mathrm{OCH} \mathrm{H}_{3}-\mathrm{Ph}$ \\
$\mathbf{5}$ & $\mathbf{1 4}$ & $\mathbf{2 3}$ & $\mathrm{CH}_{3}$ & $m-\mathrm{Ph}-\mathrm{O}-\mathrm{Ph}$ \\
$\mathbf{6}$ & $\mathbf{1 5}$ & $\mathbf{2 4}$ & $\mathrm{CH}_{3}$ & $3,5-\mathrm{OCH} \mathrm{H}_{3}-\mathrm{Ph}$ \\
$\mathbf{7}$ & $\mathbf{1 6}$ & $\mathbf{2 5}$ & $\mathrm{CH}_{2} \mathrm{CH}_{3}$ & $\mathrm{Ph}$ \\
$\mathbf{8}$ & $\mathbf{1 7}$ & $\mathbf{2 6}$ & $\mathrm{CH}_{3}$ & $m-\mathrm{Cl}-\mathrm{Ph}$ \\
$\mathbf{9}$ & $\mathbf{1 8}$ & $\mathbf{2 7}$ & $\mathrm{CH}_{2} \mathrm{CH}_{3}$ & $p-\mathrm{NO}_{2}-\mathrm{Ph}$ \\
\hline
\end{tabular}

$\& 18,27)$ and were identified as $N$-benzothiazolyl and $1(H)$ tetrahydroindazoles respectively.

Several attempts that have taken for the synthesis of $\mathrm{N}$-carbomethoxy tetrahydroindazoles under different reaction conditions were not successful. It has been concluded that condensation products were favored under alcoholic medium without acid catalyst and one of the representative compound $\mathbf{2 8}$ is described. A series of compounds synthesized by the reaction of methyl hydrazino carboxylate with the respective cylic $\beta$ keto esters in alcohol and toluene medium with the addition of acid were characterized through NMR and the resultant products were identified as $1(H)$-tetrahydroindazoles (19-27). To confirm this result, 1(H)-tetrahydroindazoles were synthesized from the respective cyclic ketoesters with hydrazine hydrate in alcoholic medium. The observed NMR results are similar to the compounds attained by using methylhydrazino carboxylate.

Formations of the $1(H)$-tetrahydroindazoles instead of the targeted molecules while using methylhydrazino carboxylate even in minimum acidity (required for the cyclization) and in higher acidity (more than the required level) in case of 2-hydrazinobenzothiazole reactions, suggest that it could be due to the cleavage of substituent linkage $\mathrm{N}-\mathrm{C}$ bond by the acid at the time of condensation between $\mathrm{NH}_{2}$ with the keto group which has been expected as first step from the plausible mechanism. ${ }^{1}$ It can be explained that the cleavage is easily ensued in methylhydrazino carboxylate even in lower acidic concentrations and not in the case of 2-hydrazinobenzothiazole is due to the variance in the sensitivity (aroused due to the polarization) of the substituent linkage $\left(\mathrm{R}-\mathrm{NHNH}_{2}\right)$ towards the acids in activated state.

Characterization of the compounds through NMR. All the synthesized compounds were characterized through NMR to find out the structure and stereochemistry without ambiguity.

Spectral analysis of compounds 19-27. Compounds 19-27 have been expected as $N$-carbomethoxy tetrahydroindazoles. The preliminary analysis of NMR showed the absence of respective resonances for the ester group at $\mathrm{C}-2$ and $\mathrm{C}-1(\mathrm{C}=\mathrm{O})$, which suggested that these groups are involved in cyclization. But, from the absence of expected resonances for carbomethoxy group and observed chemical shift pattern, it has been found that the attained compound was $1(H)$-tetrahydroindazole. The structural elucidations and stereochemistry of $1(H)$-tetrahydroindazoles, which have been synthesized from the respective reaction of dinucleophile hydrazine with cyclic $\beta$ keto esters in alcoholic medium; ${ }^{17}$ besides, one and two dimensional NMR characterization of the compound $\mathbf{1 9}$ acquired by the reaction of compound $\mathbf{1}$ with hydrazine hydrate is tabulated for reference (Supplementary material). All the compounds 19-26 synthesized by using methyl hydrazino carboxylate were found as $1(H)$-tetrahydroindazoles only. Moreover, these compounds showed similar chemical shifts for both carbons and their associated protons except for the aromatic and ester group.

Among the four doublets (each represents with one integral) observed for four cycloalkane ring protons, two doublets resonated in the range $3.98-4.07 \mathrm{ppm}$ and $2.54-2.63$ ppm having coupling constants $(J)$ around $10 \mathrm{~Hz}$ are due to the vicinal protons $\mathrm{H}-4 \mathrm{a}$ and $\mathrm{H}-5 \mathrm{a}$, in which, the highly deshielded proton observed in downfield is assigned for H-4a by considering its position, consequently the remaining was assigned for $\mathrm{H}-5 \mathrm{a}$ and another two doublets observed in the range $2.5-2.55 \mathrm{ppm}$ and $2.75-2.8 \mathrm{ppm}$ with the coupling constants around $16 \mathrm{~Hz}$ are due to the protons attached in the same carbon C-7, and the down field resonance is assigned for $\mathrm{H}-7 \mathrm{e}$ by accounting the deshielding effect aroused by the electronegativity change at $\mathrm{C}-8$ and its conformation. Methyl protons and $\mathrm{OH}$ proton at C-6 were observed at $1.19-1.23 \mathrm{ppm}$ and $4.48-4.58 \mathrm{ppm}$ respectively. A singlet observed with integral value of three in the range 3.44 - $3.47 \mathrm{ppm}$ is assigned for $\mathrm{CH}_{3}$ of $\mathrm{COOCH}_{3}$. The individual assignments have been carried out from the observed HSQC and $\mathrm{HMBC}$ correlations for compound $\mathbf{1 9}$ which are given in the supplementary data.

All the methine, methylene and methyl carbons were assigned from the respective correlations with their associated protons in HSQC spectrum observed as, 4.06/38.7 (H-4a/ C-4), 2.57/ 59.9 (H-5a/C-5), 2.52, 2.77/36.5 (H-7a, H-7e/C-7), 1.21/28.4 $\left(\mathrm{CH}_{3}\right.$ at $\left.\mathrm{C}-6\right)$ and $3.43 / 50.8\left(\mathrm{CH}_{3}\right.$ of $\left.\mathrm{COOCH}_{3}\right)$. Furthermore, the assignments of the quaternary carbons and evidence for the formation of the compounds as $1(H)$-tetrahydroindazoles and not as 2(H)-tetrahydroindazoles, have been revealed from the HMBC correlations. From the observed HMBC correlations, $138.8 \mathrm{ppm} / 2.76,2.98,4.27,1.21 \mathrm{ppm}\left(\mathrm{H}-7 \mathrm{a}, \mathrm{H}-7 \mathrm{e}, \mathrm{H}-4 \mathrm{a}, \mathrm{CH}_{3}\right.$ at C-6) and $157.9 \mathrm{ppm} / 4.27 \mathrm{ppm}(\mathrm{H}-4 \mathrm{a})$, the resonance at 138.8 ppm is conveniently assigned for C-8 and $157.9 \mathrm{ppm}$ for C-3. Such an up field resonance of C-3 carbon suggested that the 
Table 1. HSQC and HMBC Correlations of compound $\mathbf{1 1}$

\begin{tabular}{|c|c|c|}
\hline Carbon chemical shifts & HSQC Correlations & HMBC Correlations \\
\hline $152.57, \mathrm{C}-3$ & No Correlations & $4.10, \mathrm{H}-4 \mathrm{a}$ \\
\hline $37.67, \mathrm{C}-4$ & 4.10, H-4a & 7.20 (Ph-ortho 2H), 2.76 (H-5a) \\
\hline $58.91, \mathrm{C}-5$ & $2.76, \mathrm{H}-5 \mathrm{a}$ & $4.10(\mathrm{H}-4 \mathrm{a}), 2.64(\mathrm{H}-7 \mathrm{a}), 4.89(\mathrm{OH}$ at $\mathrm{C}-6), 1.28\left(\mathrm{CH}_{3}\right.$ at $\left.\mathrm{C}-6\right)$ \\
\hline $69.35, \mathrm{C}-6$ & No Correlations & $2.64(\mathrm{H}-7 \mathrm{a}), 2.99(\mathrm{H}-7 \mathrm{e}), 2.76(\mathrm{H}-5 \mathrm{a}), 4.89(\mathrm{OH}$ at C-6 $), 1.28\left(\mathrm{CH}_{3}\right.$ at $\left.\mathrm{C}-6\right)$ \\
\hline 36.96, C-7 & 2.64, H-7a; 2.99, H-7e & $2.76(\mathrm{H}-5 \mathrm{a}), 1.28\left(\mathrm{CH}_{3}\right.$ at $\left.\mathrm{C}-6\right), 4.89(\mathrm{OH}$ at C-6) \\
\hline $152.38, \mathrm{C}-8$ & No correlations & $2.64(\mathrm{H}-7 \mathrm{a}), 2.99(\mathrm{H}-7 \mathrm{e}), 4.10(\mathrm{H}-4 \mathrm{a}), 1.28\left(\mathrm{CH}_{3}\right.$ at C-6) \\
\hline $101.36, \mathrm{C}-9$ & No correlations & $4.10(\mathrm{H}-4 \mathrm{a}), 2.64(\mathrm{H}-7 \mathrm{a}), 2.99(\mathrm{H}-7 \mathrm{e})$ \\
\hline $27.93, \mathrm{CH}_{3}$ at $\mathrm{C}-6$ & $1.28\left(\mathrm{CH}_{3}\right.$ at $\left.\mathrm{C}-6\right)$ & $2.64(\mathrm{H}-7 \mathrm{a}), 4.89(\mathrm{OH}$ at $\mathrm{C}-6)$ weak \\
\hline $51.05, \mathrm{CH}_{3}$ of $\mathrm{COOCH}_{3}$ & $3.46\left(\mathrm{CH}_{3}\right.$ of $\left.\mathrm{COOCH}_{3}\right)$ & - \\
\hline $171.88, \mathrm{C}=\mathrm{O}$ of $\mathrm{COOCH}_{3}$ & No Correlations & $4.10(\mathrm{H}-4 \mathrm{a}), 2.76(\mathrm{H}-5 \mathrm{a}), 3.46\left(\mathrm{CH}_{3}\right.$ of $\left.\mathrm{COOCH}_{3}\right) 2.64(\mathrm{H}-7 \mathrm{a}$, weak $)$ \\
\hline 137.49 phenyl ipso(C-1') & No correlations & $4.10(\mathrm{H}-4 \mathrm{a}), 2.76(\mathrm{H}-5 \mathrm{a}), 7.07$, meta protons \\
\hline $114.87,114.66$ & 7.07, meta protons & - \\
\hline $129.98,129.90$ & 7.20 , ortho protons & $4.10(\mathrm{H}-4 \mathrm{a})$ \\
\hline $162.16,159.76$ & No correlations & 7.07, meta protons, 7.20 , ortho protons \\
\hline 122.12,C-d & 8.0, H-d & 7.46, H-e \\
\hline 126.40, C-e & 7.46, H-e & 8.0, H-d \\
\hline 123.87, C-f & 7.31, H-f & 7.82, H-g \\
\hline 120.50, C-g & 7.82, H-g & 7.31, H-f \\
\hline $160.1, \mathrm{C}-\mathrm{b}$ & No Correlations & 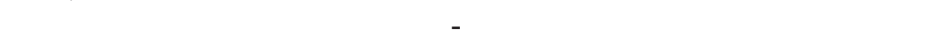 \\
\hline 148.30, C-i & No Correlations & 8.0, H-d, 7.46, H-e \\
\hline 131.69, C-h & No Correlations & 7.31, H-f, 7.82, H-g \\
\hline
\end{tabular}

(C-3) carbon involved in single bond $(\mathrm{C}-\mathrm{N})$ with the nitrogen in the fused ring confirmed the formation of the compound as 1(H)-tetrahydroindazole.

Structural elucidations of compounds 10-18. Proton NMR analysis of compounds 10-18 confirmed the formation of compounds as single isomer. Structural interpretations have been carried out by comparing with the chemical shifts of 1(H)-tetrahydroindazoles. The observed chemical shifts of both protons and carbons of cycloalkane part showed similar resonances and closer coupling constants with $1(H)$-tetrahydroindazole except for the C-8 carbon, facilitated the convenient assignments. Furthermore, the respective resonances observed for the benzothiazolyl moiety of the entire compounds showed similar chemical shifts for all the carbons and their associated protons thereby analyzed without ambiguity through the support of HSQC and HMBC recorded for the representative compound $\mathbf{1 1}$ (observed correlations are given in Table 1).

Analysis of benzothiazolyl moiety of compound 11. Among the four protons in the benzothiazolyl moiety, two protons resonated as triplets at $7.32 \mathrm{ppm}$ and $7.47 \mathrm{ppm}$ were assigned for $\mathrm{He}$ and Hf protons while another two protons resonated as doublets at $8.01 \mathrm{ppm}$ and $7.82 \mathrm{ppm}$ were assigned for $\mathrm{Hd}$ and Hf protons. Moreover, the downfield resonance at $8.01 \mathrm{ppm}$ is assigned for Hd proton, since it is having 1,3 dipolar interaction with $\mathrm{N}-\mathrm{c}$ along with extended conjucation. It has been found that in ${ }^{13} \mathrm{C}$ NMR, seven peaks were observed for benzothiazolyl group and among them three resonances are pertaining to quaternary carbons. By considering the positions and with the literature support, the resonances at $160.19 \mathrm{ppm}$ and $148.30 \mathrm{ppm}$ were assigned for $\mathrm{C}-\mathrm{b}$ and $\mathrm{C}-\mathrm{i}$ carbons respectively. From the multiple bond correlations of C-i carbon, $148.30 \mathrm{ppm} / 8.01,7.47$ ppm, it was further confirmed that these two resonances are due to $\mathrm{H}-\mathrm{d}(8.01 \mathrm{ppm})$ and $\mathrm{H}-\mathrm{e}(7.47 \mathrm{ppm})$ protons. Conse- quently from the cross peaks at 7.82, $7.32 \mathrm{ppm} / 131.66 \mathrm{ppm}$, H-f(7.82 ppm), H-g (7.32 ppm) protons and C-h (131.66 ppm) carbon were assigned.

From the respective HSQC correlations of assigned protons, H-d (8.01 ppm)/122.14 ppm (C-d), H-e (7.47 ppm)/126.43 ppm (C-e), H-f (7.82 ppm)/123.87 ppm (C-f) and H-g (7.32 ppm)/ 120.48 ppm (C-g), methine carbons were assigned.

Position of benzothiazolyl moiety. Although the observed chemical shifts of compounds $\mathbf{1 9 - 2 6}$ and $\mathbf{1 0 - 1 8}$ are almost similar (except benzothiazolyl moiety), C-3 and C-8 carbon resonances showed a considerable difference due to the position of the substituent. By accounting the upfield shift of about 5.5 ppm for C-3 carbon and downfield shift of about 14 ppm for C-8 carbon of benzothiazolyl substituted tetrahydroindazoles compared with tetrahydroindazoles concluded that the benzothiazolyl group positioned at $N(2)$. It can be described that the $\mathrm{C}=\mathrm{N}$ bond formation in compounds $\mathbf{1 0 - 1 8}$ deshields the $\mathrm{C}-8$ carbon due to the polarizability change through the double bond formation and of course the upfield shift of C-3 carbon is also due to the same.

Antimicrobial activity studies. All the synthesized compounds were screened for their in vitro antimicrobial activities against four Gram-positive bacteria viz. Bacillus subtilis (MTCC 441), Staphylococcus aureus (ATCC 25923), Staphylococcus epidermidis (MTCC 3615), Enterococcus faecalis (ATCC 29212), four Gram-negative bacteria viz. Escherichia coli (ATCC 259 22), Proteus vulgaris (MTCC 1771), Pseudomonas aeruginosa (ATCC 15380) and Klebsiella pneumoniae (ATCC 15380), and one fungal strain - Candida albicans (MTCC 227) by disc diffusion method. ${ }^{16}$ Test compounds were prepared at a concentration of $125 \mu \mathrm{g} / \mathrm{mL}$ using DMSO as solvent. Streptomycin and Chloramphenicol were used as standard drugs $(10 \mu \mathrm{g} / \mathrm{disc})$ for both antibacterial and antifungal studies. Initially, $1(H)$-tetra- 
Table 2. Antimicrobial activities of compounds 29-40 against selected microbial strains<smiles>CC1(O)Cc2nn(-c3ccccn3)c(O)c2C1C(=O)O</smiles>

\begin{tabular}{|c|c|c|c|c|c|c|c|c|c|}
\hline \multirow{4}{*}{ Entry } & \multirow{4}{*}{$\mathrm{Ar}$} & \multirow{4}{*}{$\mathrm{R}$} & & \multicolumn{6}{|c|}{ Zone of diameter of inhibition $(\mathrm{mm})^{a}$} \\
\hline & & & \multicolumn{6}{|c|}{ Antibacterial activity (in $125 \mu \mathrm{g} / \mathrm{mL}$ ) } & \multirow{3}{*}{$\begin{array}{c}\text { Antifungal activity } \\
\text { (in } 125 \mu \mathrm{g} / \mathrm{mL} \text { ) }\end{array}$} \\
\hline & & & \multicolumn{3}{|c|}{ Gram-positive } & \multicolumn{3}{|c|}{ Gram-negative } & \\
\hline & & & B.s & S. $a$ & E.f & E.c & P. $a$ & K.p & \\
\hline 29 & $\mathrm{Ph}$ & $\mathrm{Me}$ & 23 & - & 11 & - & 20 & - & 10 \\
\hline 30 & 3,5-OMe-Ph & $\mathrm{Me}$ & 22 & - & 9 & - & 18 & - & 15 \\
\hline 31 & $3-\mathrm{NO}_{2}-\mathrm{Ph}$ & $\mathrm{Me}$ & 25 & - & 10 & - & 24 & - & 16 \\
\hline 32 & 4-Cl-Ph & $\mathrm{Me}$ & 27 & - & 8 & - & 20 & - & 15 \\
\hline 33 & 3-Cl-Ph & $\mathrm{Me}$ & 16 & - & 9 & - & 15 & - & 17 \\
\hline 34 & 3-OPh-Ph & $\mathrm{Me}$ & 15 & - & 8 & - & 12 & - & 14 \\
\hline 35 & 4-Cl-Ph & $\mathrm{Et}$ & 16 & - & 9 & - & 14 & - & 15 \\
\hline 36 & 4-OMe-Ph & Et & 14 & - & - & - & 14 & - & 16 \\
\hline 37 & $4-\mathrm{NO}_{2}-\mathrm{Ph}$ & Et & 25 & - & - & - & 20 & - & 10 \\
\hline 38 & 3-Cl-Ph & Et & 25 & - & - & - & 19 & - & 9 \\
\hline 39 & 4-OMe-Ph & $\mathrm{Me}$ & 25 & - & - & - & 20 & - & 9 \\
\hline 40 & $\mathrm{Ph}$ & $\mathrm{Et}$ & 23 & - & - & - & 20 & - & 12 \\
\hline \multicolumn{2}{|c|}{ Streptomycin } & & 12 & 11 & 23 & 9 & - & 13 & - \\
\hline
\end{tabular}

$\overline{{ }^{a}}$ The data represents the mean values of two replicates. B.s: Bacillus subtilis; S.a: Staphylococcus aureus; E.f: Enterococcus faecalis; E.c: Escherichia coli: P.a: Pseudomonas aeruginosa: K.p: Klebsiella pneumoniae: C.a: Candida albicans

hydroindazole derivatives (19-27) were screened for their possible antimicrobial activities against the selected microbial organisms. Contrary to our expectation, none of the compounds exhibited inhibitory potency though the target compounds bear the biologically potent fused indazole core system.

Thus we planned to modify the target compounds in such a way to have biologically potent heterocycle at the $\mathrm{N}$ of the fused azole ring. In that view we have synthesized a series of $N(2)$-pyridyl tetrahydroindazoles (29-40) ${ }^{1}$ and explored their inhibitory activity (Table 2). To our expectation, most of the compounds exerted good inhibition potency towards the growth of Bacillus subtilis, Pseudomonas aeruginosa and Candida albicans. In particular, activity of compounds 31, 32 and 37-39 with suitably positioned chloro or nitro or methoxy functionality in the phenyl ring against Bacillus subtilis are almost twice to that of the standard drug while the noticed inhibition for the remaining compounds ranges from 14 - 23 mm (zone of inhibition). Therefore, this study clearly predicts that insertion of heterocyclic system in the indazole nucleus play a considerable role in their antimicrobial activity. However, this structural modification seems to have no significance as they failed to possess inhibitory activity against Staphylococcus aureus, Escherichia coli and Klebsiella pneumoniae.

In lieu of the biological importance of benzothiazole system, we have thus prepared the target compounds 10-18 bearing benzothizole nucleus in place of 2-pyridyl moiety and evaluated their antimicrobial activities. A close perusal of the Table 3 reveals that all the compounds exerted appreciable in vitro antimicrobial activity against the tested microorganisms. Among
Table 3. Antimicrobial activities of compounds 10-18 against selected microbial strains<smiles>CC1(O)Cc2nn(-c3nc4ccccc4s3)c(O)c2C(N)C1C(=O)O</smiles>

\begin{tabular}{|c|c|c|c|c|c|c|c|c|}
\hline \multirow{4}{*}{ Entry } & \multicolumn{8}{|c|}{ Zone of diameter of inhibition $(\mathrm{mm})^{a}$} \\
\hline & \multicolumn{7}{|c|}{ Antibacterial activity (in $125 \mu \mathrm{g} / \mathrm{mL}$ ) } & \multirow{3}{*}{$\begin{array}{c}\text { Antifungal } \\
\text { activity (in } \\
125 \mu \mathrm{g} / \mathrm{mL}) \\
\text { C.a }\end{array}$} \\
\hline & \multicolumn{3}{|c|}{ Gram-positive } & \multicolumn{4}{|c|}{ Gram-negative } & \\
\hline & B.s & S.a & S.e & P.v & E.c & P.a & K.p & \\
\hline 10 & 9 & 8 & 10 & 12 & 12 & 16 & 14 & 14 \\
\hline 11 & 8 & 12 & 13 & 10 & 14 & 11 & 13 & 13 \\
\hline 12 & 10 & 13 & 15 & 14 & 15 & 18 & 17 & 14 \\
\hline 13 & 8 & 14 & 13 & 15 & 17 & 16 & 14 & 13 \\
\hline 14 & 12 & 11 & 10 & 10 & 12 & 11 & 10 & 11 \\
\hline 15 & 11 & 15 & 16 & 12 & 14 & 16 & 14 & 10 \\
\hline 16 & 11 & 15 & 19 & 13 & 15 & 15 & 15 & 15 \\
\hline 17 & 10 & 13 & 15 & 16 & 12 & 14 & 13 & 12 \\
\hline 18 & 9 & 14 & 15 & 11 & 13 & 14 & 12 & 12 \\
\hline $\begin{array}{l}\text { Chloram- } \\
\text { phenicol }\end{array}$ & 10 & 8 & 11 & 10 & 9 & 8 & 9 & 12 \\
\hline
\end{tabular}

${ }^{a}$ The data represents the mean values of two replicates. B.s: Bacillus subtilis; S.a: Staphylococcus aureus; S.e: Staphylococcus epidermidis: P.v: Proteus vulgaris; E.c: Escherichia coli: P.a: Pseudomonas aeruginosa: K.p: Klebsiella pneumoniae: C.a: Candida albicans 
the compounds tested for their antibacterial activity, compounds 15, 16 against Staphylococcus aureus, 16 against Staphylococcus epidermidis, 13 against Escherichia coli and 10, 12,13 and 15 against Pseudomonas aeruginosa are the most efficient in inhibiting the growth of the said Gram-positive and Gram-negative bacteria. In particular, the inhibitory potency of compounds $\mathbf{1 0}$, 12, 13 and 15 against Pseudomonas aeruginosa are doubled to that of the standard chloramphenical drug. Therefore, these preliminary results clearly indicate that the presence of methyl ester instead of its ethyl counterpart seems to be favorable in eliciting the better antimicrobial responses besides the presence of either chlorine or methoxy group at the para position of the phenyl group. As well, of the two heterocyclic systems introduced at the 2 nd position of the indazole system, benzothiazole nucleus is promising in exerting significant inhibitory responses towards all the tested pathogenic microbial strains than the 2-pyridyl system.

According to structure-activity relationship (SAR), it can be concluded that presence of benzothiaole moiety is essential for the antimicrobial activity in addition to the presence of either electron donating or withdrawing functionality at the para position of phenyl group.

\section{Conclusion}

Cyclic $\beta$ ketoesters have been used as a versatile synthons for the stereospecific synthesis of $N$-benzothiazolyl tetrahydroindazoles. Acidity of the reaction medium played an important role for the cyclization and in situ cleavage of the hydrazine reagents which leads to the formation of $1(H)$-indazoles. It has been found by one and two dimensional NMR investigations that the position of benzothiazolyl moiety in the indazole ring is at $N(2)$. Antimicrobial studies of the compounds against the selected bacteria and fungus concluded that $1(H)$-tetrahydroindazoles did not show the expected potency. But, benzothiazolyl substituted indazoles exerted remarkable enhancement in the activity and are even by far better than the potency exhibited by the corresponding pyridyl analogues.

Supplementary Information Available. HSQC and HMBC correlations of compound $\mathbf{2 8}$ are available as an attachment.

Acknowledgments. This study was financially supported by Pukyong National University in the 2009 Post-Doc. Program.

\section{References}

1. Amirthaganesan, S.; Aridoss, G.; Park, Y. H.; Kim, J. S.; Son, S. M.; Jeong, Y. T. Heterocycles 2008, 75, 537.

2. (a) Katritzky, A. R.; Wang, M.; Zhang, S.; Voronkov, M. V. J. Org. Chem. 2001, 66, 6787. (b) Elguero, J., Katritzky, A. R., Rees, C. W., Scriven, E. F., Eds.; In Comprehensive Heterocyclic Chemistry II; Elsevier: Oxford, 1996; p 1. (C) Cho, C. S.; Patel, D. B. Tetrahedron 2006, 62, 6388. (d) Wang, X-j.; Tan, J.; Grozinger, K.; Betageri, R.; Kirrane, T.; Proudfoot, J. R. Tetrahedron Lett. 2000, 41, 5321 .

3. (a) Machado, P.; Rosa, F. A.; Rossatto, M.; Sant'Anna, G. S.; Sauzem, P. D.; Siqueira da Silva, R. M.; Rubin, M A.; Ferreira, J.; Bonacorso, H. G.; Zanatta, N.; Martins, M. A. P. Arkivoc 2007, $x v i$, 281. (b) Genin, M. J.; Biles, C.; Keiser, B. J.; Poppe, S. M.; Swaney, S. M.; Tarpley, W. G.; Yagi, Y.; Romero, D. L. J. Med. Chem. 2000, 43, 1034. (c) Stauffer, S. R.; Coletta, C. J.; Tedesco,
R.; Nishiguchi, G.; Carlson, K.; Sun, J.; Katzenellenbogen, B. S.; Katzenellenbogen, J. A. J. Med. Chem. 2000, 43, 4934. (d) Bailey, D. M.; Hansen, P. E.; Hlavac, A. G.; Baizman, E. R.; Pearl, J.; Defelice, A. F.; Feigenson, M. E. J. Med. Chem. 1985, 28, 256.

4. (a) Lukin, K.; Hsu, M. C.; Fernando, D.; Leanna, M. R. J. Org. Chem. 2006, 71, 8166. (b) Cadogan, J. I. G.; Cameron-Wood, M.; Mackie, R. K.; Searle, R. J. G. J. Chem. Soc. 1965, 483. (c) Song, J. J.; Yee, N. K. Org. Lett. 2000, 2, 519. (d) Lam, P. Y. S.; Clark, C. G.; Saubern, S.; Adams, J.; Winters, M. P.; Chan, D. M. T.; Combs, A. Tetrahedron Lett. 1998, 39, 2941. (e) Mills, A. D.; Nazer, M. Z.; Haddadin, M. F. J.; Kurth, M. J. J. Org. Chem. 2006, 71, 2687.

5. (a) Claramunt, R. M.; Lopez, C.; Perez-Medina, C.; Pinilla, E.; Torres, M. R.; Elguero, J. Tetrahedron 2006, 62, 11704 . (b) Meegalla, S. K.; Doller, D.; Liu, R.; Sha, D.; Soll, R. M.; Dhanoa, D. S. Tetrahedron Lett. 2002, 43, 8639.

6. (a) Wiener, J. J. M.; Gomez, L.; Venkatesan, H.; Santillán, A., Jr.; Allison, B. D.; Schwarz, K. L.; Shinde, S.; Tang L Hack, M. D.; Morrow, B. J.; Motley, S. T.; Goldschmidt, R. M.; Shaw, K. J.; Jones, T. K.; Grice, C. A. Bioorg. Med. Chem. Lett. 2007, 17, 2718. (b) Rodgers, J. D.; Johnson, B. L.; Wang, H.; Greenberg, R. A.; Erickson-Viitanen, S.; Klabe, R. M.; Cordova, B. C.; Rayner, M. M.; Lam, G. N.; Chang, C. H. Bioorg. Med. Chem. Lett. 1996, 6, 2919. (c) Corsi, G.; Palazzo, G.; Germani, C.; Barcellona, P. S.; Silvestrini, B. J. Med. Chem. 1976, 19, 778. (d) Bermudez, J.; Fake, C. S.; Joiner, G. F.; Joiner, K. A.; King, F. D.; Miner, W. D.; Sanger, G. J. J. Med. Chem. 1990, 33, 1924.

7. (a) Angelis, M. D.; Stossi, F.; Carlson, K. A.; Katzenellenbogen, B. S.; Katzenellenbogen, J. A. J. Med. Chem. 2005, 48, 1132. (b) May, J. A.; Dantanarayana, A. P.; Zinke, P. W.; McLaughlin, M. A.; Sharif, N. A. J. Med. Chem. 2006, 49, 318.

8. (a) Foloppe, N.; Fisher, L. M.; Francis, G.; Howes, R.; Kierstan, P.; Potter, A. Bioorg. Med. Chem. 2006, 14, 1792. (b) Cerecetto, H.; Gerpe, A.; Gonzalez, M.; Aran, V. J.; Ochoa de Ocariz, C. Mini-Rev. Med. Chem. 2005, 5, 869.

9. (a) Kirsche, K., Schaumann, E., Eds.; Methoden der Organische Chemie (Houben-Weyl); Georg Thieme: Stuttgart, 1994; p 399. (b) Elguero, J.; Fruchier, A.; Jagerovic, N.; Werner, Org. Prep. Proc. Int. 1995, 27, 33.

10. (a) Nagakura, M.; Tomio, O.; Shimidzu, S.; Eto, L. Y.; Wada, Y. J. Med. Chem. 1979, 22(1), 48. (b) Connolly, P. J.; Westin, C. D.; Loughney, D. A.; Minor, L. K. J. Med. Chem. 1993, 36, 3674. (c) Connolly, P. J.; Wetter, S. K.; Beers, K. N.; Hamel, S. C.; Johnson, D. H.; Pat Kraft, M. K.; Lai, M. T.; Campen, C.; Palmer, S.; Phillips, A. Bioorg. Med. Chem. Lett. 1997, 7(1), 2551. (d) Rosati, O.; Curini, M.; Marcotullio, M. C.; Macchiarulo, A.; Perfumi, M.; Mattioli, L.; Rismondo, F.; Cravottoc, G. Bioorg. Med. Chem. 2007, 15, 3463. (e) Lee, K. Y.; Gowrisankar, S.; Kim, J. N. Tetrahedron Lett. 2005, 46, 5387. (f) Dvorak, C. A.; Rudolph, D. A.; Ma, S.; Carruthers, N. I. J. Org. Chem. 2005, 70, 4188. (f) Lee, K. Y.; Kim, J. M.; Kim, J. N. Tetrahedron Lett. 2003, 44, 6737.

11. Minkin, V. I.; Garnosvskii, A. D.; Elguero, J.; Katritzky, V.; Denisko, O. V. Adv. Heterocycl. Chem. 2000, 76, 157-323.

12. (a) Katritzky, A. R. Advances in Heterocyclic Chemistry; Academic Press: 1994; p 60. (b) Bouabdallah, I.; Touzani, R.; Zidane, I.; Ramdani, A.; Radia, S. Arkivoc 2006, xii, 138. (d) Albar, H. A. J. Chem. Research (S) 1999, 182.

13. (a) Lopez-Alvarado, P.; Avendano, C.; Menendez, J. C. J. Org. Chem. 1995, 60, 5678. (b) Lam, P. Y. S.; Clark, C. G.; Saubern, S.; Adams, J.; Winters, M. P.; Chan, D. M. T.; Combs, A. Tetrahedron Lett. 1998, 39, 2941. (c) Fedorov, A. Yu.; Finet, J. Tetrahedron Lett. 1999, 2747.

14. Claramunt, R. M.; Elguero, J.; Garceran, R. Heterocycles $\mathbf{1 9 8 5}$ 2895.

15. Pandiarajan, K.; Sabapathy Mohan, R. T.; Gomathi, R.; Muthukumaran, G. Magn. Reson. Chem. 2005, 43, 430.

16. Murray, P. R.; Baron, E. J.; Pfaller, M. A.; Tenover, F. C.; Yolke, R. H. In Manual of Clinical Microbiology; Vol. 6. ASM: Washington DC, 1995.

17. Amirthaganesan, S. Ph. D., Thesis, Annamalai University, India, 2005. 